Research Article

\title{
Comparative Study between Pars Plana Vitrectomy with Internal Limiting Membrane Peel and Pars Plana Vitrectomy with Internal Limiting Membrane Flap Technique for Management of Traumatic Full Thickness Macular Holes
}

\author{
Hammouda Hamdy Ghoraba, ${ }^{1,2}$ Mahmoud Leila $\mathbb{D}^{3},{ }^{3}$ Hashem Ghoraba $\mathbb{D}^{4},{ }^{4}$ \\ Mohamed Amin Heikal (D, ${ }^{5}$ and Emad Eldin Mohamed Elgemai ${ }^{6,7}$ \\ ${ }^{1}$ Professor of Ophthalmology, Faculty of Medicine, Tanta University, Tanta, Egypt \\ ${ }^{2}$ Medical Director, Magrabi Eye Hospital, Tanta, Egypt \\ ${ }^{3}$ Associate Professor of Ophthalmology, Retina Department, Research Institute of Ophthalmology, Giza, Egypt \\ ${ }^{4}$ Ophthalmology Specialist, Magrabi Eye Hospital, Tanta, Egypt \\ ${ }^{5}$ Assistant Professor of Ophthalmology, Faculty of Medicine, Benha University, Benha, Egypt \\ ${ }^{6}$ Ophthalmology Consultant, Damanhour Teaching Hospital, Damanhour, Egypt \\ ${ }^{7}$ Ophthalmology Consultant, Magrabi Eye Hospital, Tanta, Egypt \\ Correspondence should be addressed to Mahmoud Leila; mahmoudleila@yahoo.com
}

Received 20 December 2018; Revised 23 February 2019; Accepted 10 March 2019; Published 21 April 2019

Guest Editor: Akihiro Ohira

Copyright (c) 2019 Hammouda Hamdy Ghoraba et al. This is an open access article distributed under the Creative Commons Attribution License, which permits unrestricted use, distribution, and reproduction in any medium, provided the original work is properly cited.

\begin{abstract}
Purpose. To compare the efficacy of PPV and ILM peel versus PPV and IFT in patients with traumatic FTMH. Methods. Retrospective interventional comparative case series including two groups of patients with traumatic FTMH. Patients were divided into group I (ILM peel) and group II (IFT). The main outcome measure was closure of the macular hole and restoration of the foveal microstructure. The independent-samples T-test and ANOVA test were used to study the mean between 2 groups and calculate the $P$ value, whereas the bivariate correlation procedure studied the interaction between the variables tested. Results. Group I included 28 patients. Mean preoperative MLD was $757 \mu \mathrm{m}$. Mean preoperative BCVA was approximately 20/320. Group II included 12 patients. Mean preoperative MLD was $529.5 \mu \mathrm{m}$. Mean preoperative BCVA was 20/320. Group I had a macular hole closure rate of $75 \%$ versus $92 \%$ in group II $P=0.05$. Mean BCVA improvement was 2.5 lines in group I versus 5 lines in group II $P=0.02$. Disrupted ELM and IS/OS was the most salient finding in both groups. Conclusion. IFT has a significantly superior anatomic and functional outcome compared to ILM peel in traumatic FTMH.
\end{abstract}

\section{Introduction}

The key success of pars plana vitrectomy (PPV) and gas tamponade for FTMH introduced by Kelly and Wendel $[1,2]$ was attributed to counteracting the anteroposterior vitreous traction at the perifoveal area. On the contrary, the fluid-gas exchange flattened the subretinal fluid cuff surrounding the hole, and the gas bubble then prevented fluid currents from interfering with the healing process [3-7]. The introduction of internal limiting membrane (ILM) maculorhexis was a significant rider to the original technique that greatly spurred the success rates of surgery, although with better response in smaller size holes compared to larger size holes $>400 \mu \mathrm{m}$ in diameter [8-10]. Peeling off the ILM from the vicinity of a FTMH had dual benefit. Firstly, it eliminated the tangential forces created by glial cells migrating through ILM microrips. Secondly, it induced shearing of the Müller cells' foot plates thereby triggering glial cells proliferation along the interface created by the gas bubble, eventually inducing closure of the hole [11]. While 
this scenario applied to primary FTMH that were caused by anomalous vitreofoveolar traction, similar success was not achieved in FTMH secondary to trauma $[12,13]$. The pathogenic mechanisms entailed in traumatic FTMH formation included direct injury from blunt trauma inducing the classic trampoline effect or from open globe injury, and indirect injury from a propagating shock wave of chorioretinitis sclopetaria or pressure necrosis of the foveal area by subfoveal hemorrhage [14-17]. These mechanisms steered the pathological cascade to a common endpoint, which is hole formation due to tissue loss. This meant posing a significant pathological element that could not be rectified by the aforementioned surgical maneuvers and that hindered anatomical closure and retinal layers' structural recovery. Accordingly, traumatic FTMH acquired notoriety of frequent initial failure, late reopening, and worse final visual outcome compared to the primary variant $[18,19]$. The aim of the current study is to compare the efficacy of PPV and ILM peel versus PPV and ILM flap technique (IFT) in terms of anatomic and functional outcomes in patients with traumatic FTMH.

\section{Patients and Methods}

This was a retrospective interventional comparative case series that analyzed data of 40 consecutive patients with traumatic FTMH, who were treated in a private ophthalmic center, Magrabi Eye Hospital, Tanta, Egypt, over the past 5 years. Prior to 2017, our surgical protocol for treating traumatic holes consisted of PPV and ILM peel. As from 2017, all patients underwent PPV and IFT. Preoperative data included age, gender, eye involved, type of trauma, and duration of the disease. Best-corrected visual acuity (BCVA) was measured using the Snellen notation and converted to logarithm of minimum angle of resolution (logMAR) for statistical analysis. Diagnosis of FTMH was based on biomicroscopic examination and optical coherence tomography (OCT) imaging (Cirrus HD-OCT 4000 (Carl Zeiss Meditech, Inc., Dublin, California, USA)) or Heidelberg Spectralis Spectral-Domain OCT (SD-OCT (Heidelberg Engineering, Inc., Heidelberg, Germany)), using highdefinition 5-line raster scans and 3-dimensional 512 $\times 128$ macular cube scans passing through the fovea. Biomicroscopically, an FTMH was defined as a central round retinal defect with a rim of elevated retina. Weiss's ring and/ or prefoveolar opacity may be present or absent. The size of the central retinal defect was calibrated against the diameter of one of the large tributaries of the central retinal vein close to the optic disc margin [3]. On OCT imaging, an FTMH was defined as an anatomic defect in the fovea involving all neural layers from the ILM to the retinal pigment epithelium (RPE) detected on at least one OCT B-scan. The size of the hole was assessed using the minimum linear diameter (MLD), which was measured using software built-in calipers feature. The MLD was determined by drawing a horizontal line parallel to the RPE between the narrowest hole points in the midretina, i.e., at the shortest distance across the full thickness defect [20]. The study recruited exclusively patients with naïve FTMH with unequivocal history of blunt ocular trauma. Exclusion criteria included recurrent or persistent macular holes following previous surgery, associated retinal detachment or proliferative vitreoretinopathy, significant corneal opacity that would hinder ILM surgery, associated consecutive optic atrophy due to traumatic optic neuropathy, or a follow-up duration less than 3 months. Recruited patients were classified into two treatment arms. Group I included patients who underwent PPV with ILM peel, whereas group II included patients who underwent PPV with IFT. The main outcome measure was closure of the macular hole and restoration of foveal microstructure. Secondary outcome measures were correlation between preoperative MLD, baseline BCVA, duration of the hole prior to surgical intervention, and the anatomical outcome (type of closure and status of foveal microstructure) and the functional outcome (postoperative BCVA), in addition to development of complications. Selection of patients for enrollment in the study and all surgical procedures entailed were undertaken by single vitreoretinal surgeon (HG). The current study was approved by the Institutional Review Board of Magrabi Eye Hospital in Egypt. The study adhered strictly to the tenets of the Declaration of Helsinki (2013 revision). All individuals enrolled in the study received thorough explanation of the surgical procedures entailed, the expected outcomes and possible complications. Afterwards, they were asked to sign an informed consent before undertaking surgery. The consent included a statement that authorized the authors to publish patients' data for research purposes in an anonymous manner that does not allow identification of the patient.

2.1. Surgical Technique. Recruited patients who presented with concomitant significant cataract that could hinder adequate visualization during PPV and ILM manipulation underwent PPV combined with standard phacoemulsification with foldable intraocular lens implantation within the capsular bag. Surgical technique in all cases consisted of 23-gauge 3-port PPV, followed by triamcinolone acetonide- (TA-) assisted induction of posterior vitreous detachment (PVD) that was accomplished by applying aspiration over the optic nerve head $(\mathrm{ONH})$ using the vitreous cutter. Once induced, PVD was carried up to the equator. We routinely used TA for ILM peel. After PVD induction, $0.2 \mathrm{ml}$ TA suspension was sprayed onto the macular area. The supernatant suspension is aspirated from the vitreous cavity while allowing large-sized TA particles to settle down over the ILM. Peeling was started by directly pinching the ILM at a point of natural weakness over the inferior temporal arcade using a 23-gauge Eckardt end-gripping forceps (D.O.R.C. Dutch Ophthalmic Research Center (International) B.V., Netherlands). Once a flap is created, it is slightly elevated over the retinal surface then ripped tangentially in a rhexis fashion for at least 2 disc diameters (DD) from the hole. ILM peeling maneuver was performed centripetal to the fovea to avoid enlargement of the hole. During ILM removal, the peeled ILM flap with overlying TA particles was easily identified from the unpeeled ILM. Additional clues for ILM identification included its peculiar glistening reflex, which provided clear contrast with the dullwhite appearance of the denuded retina in the peeled area, 
and/or petechial surface hemorrhages in the peeled area. In cases with inadequate visualization of the ILM, we resorted to ILM-blue stain (D.O.R.C. Dutch Ophthalmic Research Center (International) B.V., Netherlands).

In group I, the ILM was completely removed off the macular hole, whereas in group II, the ILM peel was stopped at the edges of the macular hole forming an island of ILM floating into the vitreous cavity with a $360^{\circ}$ attachment to the hole. Redundant peripheral edges of the flap were trimmed by the vitreous cutter using shaving mode with minimal suction. No attempts were made to fold over, dip, or tuck the flap inside the hole to avoid traumatizing the RPE. The retinal periphery was then inspected by scleral depression to check for iatrogenic holes, followed by fluid-air exchange. After removal of the 3 cannulas air/C2F6, hexafluoroethane gas exchange was performed using two 30-gauge needles, one for injection of $14 \%$ C2F6 and the other for simultaneous air venting (Supplemental digital content, video 1 demonstrates the IFT using TA). Postoperatively, patients were asked to adopt a drinking-bird positioning protocol, in which the patient had to maintain a face-down posture every other 15 minutes for $50 \%$ of his/her waking time for 1 week or until $50 \%$ of the gas was absorbed as judged by biomicroscopic examination.

2.2. Postoperative Follow-Up. During the postoperative period, patients were examined at 1 day, 1 week, 1 month, and 3 months thereafter. Postoperative data included BCVA, intraocular pressure (IOP) measurement, assessment of macular hole closure biomicroscopically and on OCT examination, and development of postoperative complications.

2.2.1. Macular Hole Closure. On biomicroscopy, macular hole closure was defined as complete apposition of the hole margins and restoration of the foveal light reflex. Patients were then classified according to the closure type and restoration of foveal microstructure on OCT imaging as follows.

(1) Closure Type. U-type configuration was defined as closed macular hole with normal foveal contour; V-type configuration was defined as closed macular hole with steep foveal contour, whereas W-type configuration was considered an open flat macular hole with persistent neurosensory retinal defect [21].

(2) Foveal Microstructure. Category 1 included eyes with restored external limiting membrane (ELM) and inner segment/outer segment (IS/OS) junction; category 2 included eyes with restored ELM and disrupted IS/OS junction, whereas category 3 included eyes with disrupted both ELM and IS/OS junction. Category 4 included eyes with persistent open hole after surgery.

\subsection{Statistical Analysis}

2.3.1. Independent-Samples T-Test. The independentsamples $T$-test procedure compares means for two groups of cases. Ideally, for this test, the subjects should be randomly assigned to two groups, so that any difference in response is due to the treatment and not due to other factors. For each variable, sample size, mean, standard deviation, and standard error of the mean were calculated. For the difference in means, mean and standard error were calculated. The significance of the measured $T$-test value was considered as follows: not significant (NS) when $P>0.05$, significant (S) when $P \leq 0.05\left({ }^{*}\right)$, highly significant (HS) when $P<0.01\left({ }^{* *}\right)$, and very highly significant (V.H.S.) when $P<0.001\left(^{* * *}\right)$, where $P$ is the probability (reflect of null hypothesis).

2.3.2. Analysis of Variance (ANOVA): (F-Test). ANOVA is a procedure used for testing the differences among the means of two or more treatments. It was noted that if means of subgroups are greatly different, the variance of the combined groups is much larger than the variance of the separate groups. The ANOVA format for the analysis of differences in means is based on this fact.

2.3.3. Correlation Matrix. The bivariate correlation procedure computes Pearson's correlation coefficient that measures how variables are related. Two variables can be perfectly related, but if the relationship is not linear, Pearson's correlation coefficient is not an appropriate statistic. The results of $r$ value were checked on $r$ table to find out the significant level.

\section{Results}

3.1. Baseline Patients' Characteristics. The study included 40 eyes of 40 patients divided in 2 groups. Group I included 28 patients (23 males and 5 females), with a mean age of 21.4 years (range: $5-54$ years; SD 13). Mean preoperative MLD was $757 \mu \mathrm{m}$ (range: $412 \mu \mathrm{m}-1200 \mu \mathrm{m}$; SD 221). Mean preoperative BCVA was $1.25 \log$ MAR (Snellen equivalent approximately 20/320) (range: $1.5-0.7 \operatorname{logMAR}$; SD 0.3). Mean duration prior to surgical intervention was 9 months (range: 0.06-120 months; SD 23.5). Mean duration of follow-up after surgery was 37 months (range: $3-171$ months; SD 45). Group II included 12 male patients, with a mean age of 18 years (range: 7-30 years; SD 7). Mean preoperative MLD was 529.5 $\mu \mathrm{m}$ (range: $225 \mu \mathrm{m}-808 \mu \mathrm{m}$; SD 148). Mean preoperative BCVA was 1.2 logMAR (Snellen equivalent approximately 20/ 320) (range: $1.5-0.6 \log M A R ; S D ~ 0.3)$. Mean duration prior to surgical intervention was 5 months (range: $0.3-12$ months; SD 4.5). Mean duration of follow-up after surgery was 6.3 months (range: 3-12 months; SD 3.3). Statistical analysis revealed no significant difference between both groups in terms of preoperative BCVA and duration prior to surgical intervention, $(P=0.5)$, although both groups differed significantly in terms of preoperative MLD and duration of follow-up after surgery, $(P=0.003$ and 0.025 , respectively). Baseline patients' characteristics of each group are summarized in Table 1.

\subsection{Postoperative Anatomical Outcome}

3.2.1. Closure Type. Postoperatively, V-type configuration was the main closure type detected in group I, as it occurred 
TABLE 1: Baseline patients' characteristics.

\begin{tabular}{|c|c|c|}
\hline Baseline characteristics & $\begin{array}{c}\text { Group I } \\
N=28\end{array}$ & $\begin{array}{c}\text { Group II } \\
N=12\end{array}$ \\
\hline \multicolumn{3}{|l|}{ Age (years) } \\
\hline Mean & 21.4 & 18 \\
\hline$<20$ & $16(57 \%)$ & $7(58 \%)$ \\
\hline $20-40$ & $9(32 \%)$ & $5(42 \%)$ \\
\hline$>40$ & $3(11 \%)$ & - \\
\hline \multicolumn{3}{|l|}{ Gender } \\
\hline Male & $23(82 \%)$ & $12(100 \%)$ \\
\hline Female & $5(18 \%)$ & - \\
\hline \multicolumn{3}{|l|}{ BCVA logMAR (Snellen) } \\
\hline Mean & $1.25(\sim 20 / 320)$ & $1.2(20 / 320)$ \\
\hline$>1(<20 / 200)$ & $19(68 \%)$ & $8(67 \%)$ \\
\hline $1-0.7(20 / 100-20 / 200)$ & $9(32 \%)$ & $2(17 \%)$ \\
\hline$<0.7(>20 / 100)$ & - & $2(17 \%)$ \\
\hline \multicolumn{3}{|l|}{$\operatorname{MLD}(\mu \mathrm{m})$} \\
\hline Mean & 757 & 529.5 \\
\hline$<400$ & - & $1(8.3 \%)$ \\
\hline $400-600$ & $9(32 \%)$ & $8(67 \%)$ \\
\hline $601-800$ & $5(18 \%)$ & $2(17 \%)$ \\
\hline$>800$ & $14(50 \%)$ & $1(8.3 \%)$ \\
\hline \multicolumn{3}{|l|}{ Disease duration (months) } \\
\hline Mean & 9 & 5 \\
\hline$<1$ & $12(43 \%)$ & $2(17 \%)$ \\
\hline $1-6$ & $10(36 \%)$ & $6(50 \%)$ \\
\hline$>6-12$ & $2(7 \%)$ & $4(33.3 \%)$ \\
\hline$>12$ & $4(14 \%)$ & - \\
\hline \multicolumn{3}{|l|}{ Follow-up (months) } \\
\hline Mean & 37 & 6.3 \\
\hline $3-6$ & $11(39 \%)$ & $7(58 \%)$ \\
\hline$>6-12$ & $5(18 \%)$ & $5(42 \%)$ \\
\hline$>12$ & $12(43 \%)$ & - \\
\hline
\end{tabular}

BCVA, best-corrected visual acuity; logMAR, logarithm of the minimal angle of resolution; $\mu \mathrm{m}$, micron; MLD, minimum linear diameter.

in 15 eyes (53.5\%), W-type configuration occurred in 7 eyes (25\%), whereas U-type configuration was detected in 6 eyes (21.4\%). In group II, U-type configuration was detected in 6 eyes (50\%), V-type configuration was detected in 5 eyes (42\%), and W-type configuration was detected in 1 eye (8.3\%) (Figures 1-3).

3.2.2. Foveal Microstructure. In group I, 3 eyes (11\%) had restored ELM and IS/OS, 5 eyes (18\%) had restored ELM and disrupted IS/OS, 13 eyes (46.4\%) had disrupted ELM and IS/ OS, and 7 eyes (25\%) had persistent open holes. In group II, failure of restoration of both layers was detected in 10 eyes (83.3\%). Restoration of ELM and IS/OS and persistent open hole were detected in 1 eye $(8.3 \%)$ each. Therefore, disrupted ELM and IS/OS was the most salient finding in both groups.

3.3. Postoperative Functional Outcome. At the end of followup, mean BCVA in group I was $1 \operatorname{logMAR}$ (Snellen equivalent 20/200) (range: 1.5-0.2 logMAR; SD 0.4). Mean improvement was 2.5 lines. Three patients (11\%) had final BCVA 0.3 logMAR or better (Snellen equivalent $\geq 20 / 40$ ). On the contrary, mean BCVA in group II was $0.7 \log \mathrm{MAR}$
(Snellen equivalent 20/100) (range: 1.3-0.4 logMAR; SD 0.2). Mean improvement was 5 lines. No patient in group II achieved final BCVA $0.3 \log$ MAR or better.

3.4. Complications. Cataract formation or progression of incipient cataract that required cataract surgery during the course of follow-up occurred in 12 eyes (43\%) in group I and in 3 eyes (25\%) in group II. Postoperative anatomical and functional outcomes of each group are summarized in Table 2.

\subsection{Statistical Correlation of Studied Parameters}

3.5.1. Surgical Technique: PPV with ILM Peel versus PPV with IFT. Statistical analysis revealed significant correlation between IFT, better postoperative BCVA, and more favorable closure type of macular hole compared to ILM peel, $p 0.02$ and $p 0.05$, respectively. Failure of restoration of foveal microstructure was the most common outcome of both techniques $(64.4 \%$ vs. $83.3 \%$ in ILM peel and IFT, respectively) (Table 3 ).

3.5.2. Correlation between Preoperative Parameters and Anatomical Outcome (Type of Macular Hole Closure and Foveal Microstructure). Statistical analysis in group I and group II, in terms of correlation between preoperative MLD and disease duration prior to surgical intervention versus type of macular hole closure and the degree of restoration of foveal microstructure whether absent, partial, or complete, revealed no statistical significance between these variables.

3.5.3. Correlation between Preoperative Parameters and Final $B C V A$. Statistical analysis revealed that preoperative BCVA and preoperative MLD were statistically significant parameters influencing the postoperative BCVA in group I patients only, $p 0.03$ and $p 0.004$, respectively. Conversely, duration of disease prior to surgical intervention was not a significant factor influencing postoperative BCVA in both groups (Tables 4 and 5).

\section{Discussion}

In the present study, we report our experience in using PPV and ILM peel versus PPV and IFT for management of traumatic FTMH. Analysis of the anatomical and functional outcomes in group I revealed macular hole closure rate of $75 \%$, of which $21.4 \%$ was U-type. BCVA improved by a mean of 2.5 lines. Three patients (11\%) had final BCVA $0.3 \log$ MAR or better (Snellen $\geq 20 / 40$ ). In group II, the macular hole closure rate was $92 \%$, of which $50 \%$ was U-type. BCVA improved by a mean of 5 lines. In comparison, Kuhn et al. [22] reported 17 eyes with traumatic macular hole that were treated with PPV and ILM peel. The authors had macular hole closure rate of $100 \%$ and improvement of BCVA $\geq 2$ lines in $94 \%$ of eyes. It is worthy of note that all eyes in that series had either stage 2 or stage 3 holes at presentation, in comparison to the present study in 


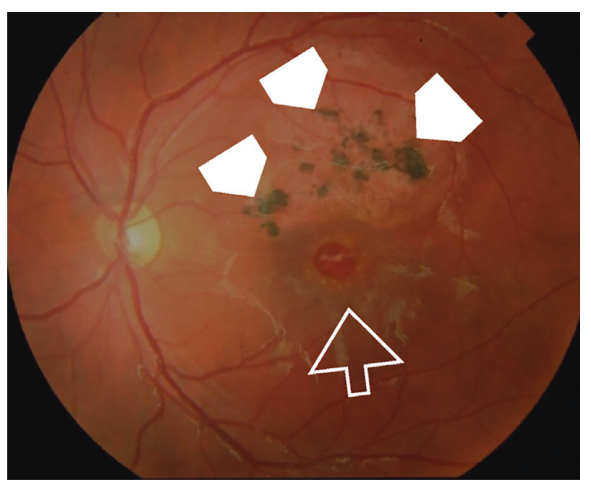

(a)

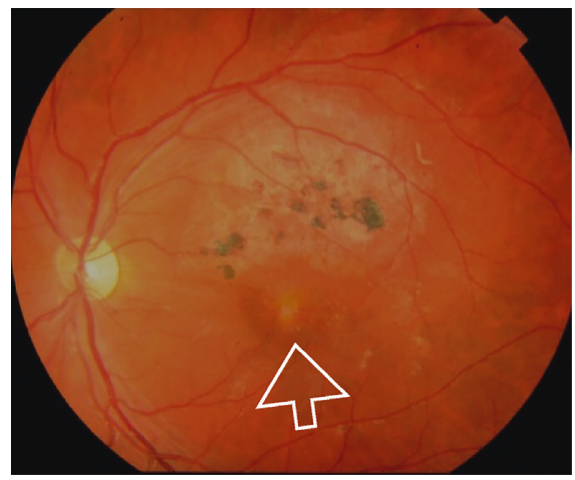

(c)

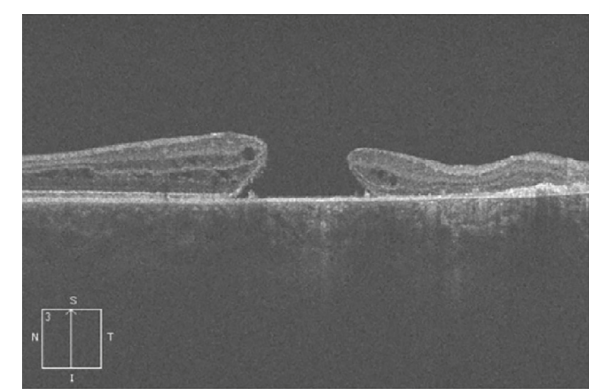

(b)

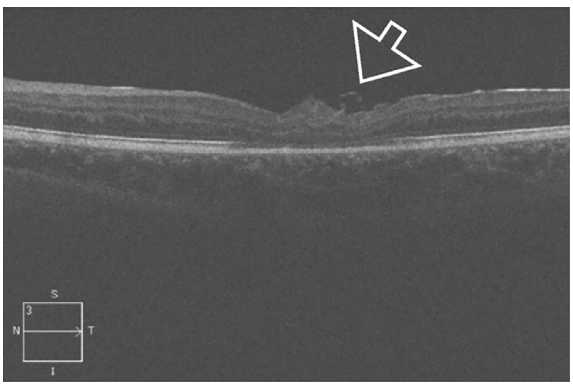

(d)

Figure 1: (a) Color photo of the left eye of a 16-year-old male patient. The patient sustained blunt trauma to the left eye with a brick approximately 12 months earlier. His BCVA was 0.05 Snellen. The posterior pole showed a large FTMH approximately $2 / 3$ DD (white arrow). Note the area of diffuse RPE mottling with RPE pigment clumps in the superior vicinity of the hole denoting the chronic course (white arrowheads). (b) High-definition 5-line raster OCT image of the same eye showed large FTMH with MLD $808 \mu \mathrm{m}$. Note the cystic thickening at the edges of the hole. (c) Color photo of the same eye approximately 6 weeks after PPV and IFT, showing successful hole closure (white arrow). His final BCVA was 0.2 Snellen. (d) High-definition 5-line raster OCT image postoperatively showing U-type closure. The ELM and IS/OS were not restored. Note the curled edge of the ILM flap (white arrow).

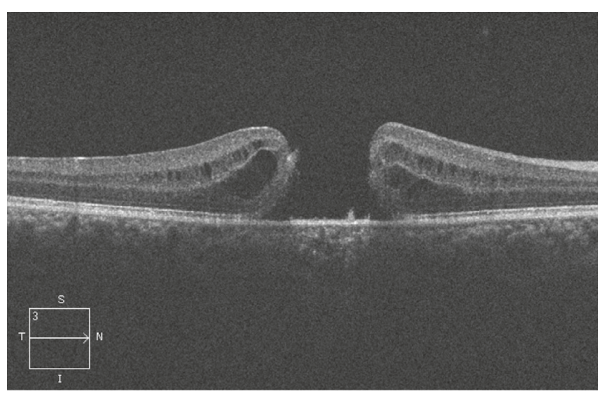

(a)

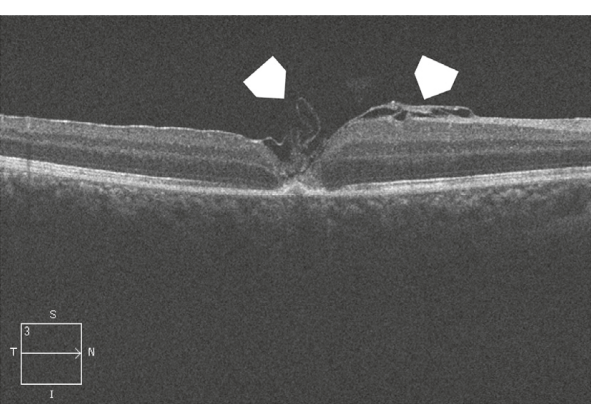

(b)

FIgURE 2: (a) High-definition 5-line raster OCT image of the right eye of a 14-year-old male. The patient was hit by a tennis ball at 1.5 months. The resultant FTMH had MLD $725 \mu \mathrm{m}$. His BCVA was 0.05 Snellen. Note the cystic thickening at the edges of the hole. (b) Highdefinition 5-line raster OCT image of the same eye 2 months after PPV and IFT showing V-type closure. The ELM and IS/OS were not restored. The free ILM flap crumbled into the macular hole (white arrowheads). His final BCVA was 0.4 Snellen.

which the MLD was $757 \mu \mathrm{m}$ and $529.5 \mu \mathrm{m}$ in groups I and II, respectively. Johnson et al. [14] studied retrospectively 25 eyes with traumatic macular holes and reported macular hole closure rate of $96 \%$ and mean improvement of BCVA $\geq 2$ lines in $84 \%$ of cases. Twenty-one patients (84\%) had stage 2 or 3 holes. ILM was peeled in only 3 cases, and autologous serum was used in $48 \%$ of cases. Weichel and Colyer [15] reported macular hole closure rate of $67 \%$. The authors included eyes with both closed and open globe injuries. ILM peel was not done in any case. They did not comment on the size of the macular hole at presentation. A case series by $\mathrm{Ou}$ et al. [17] included 5 patients with 


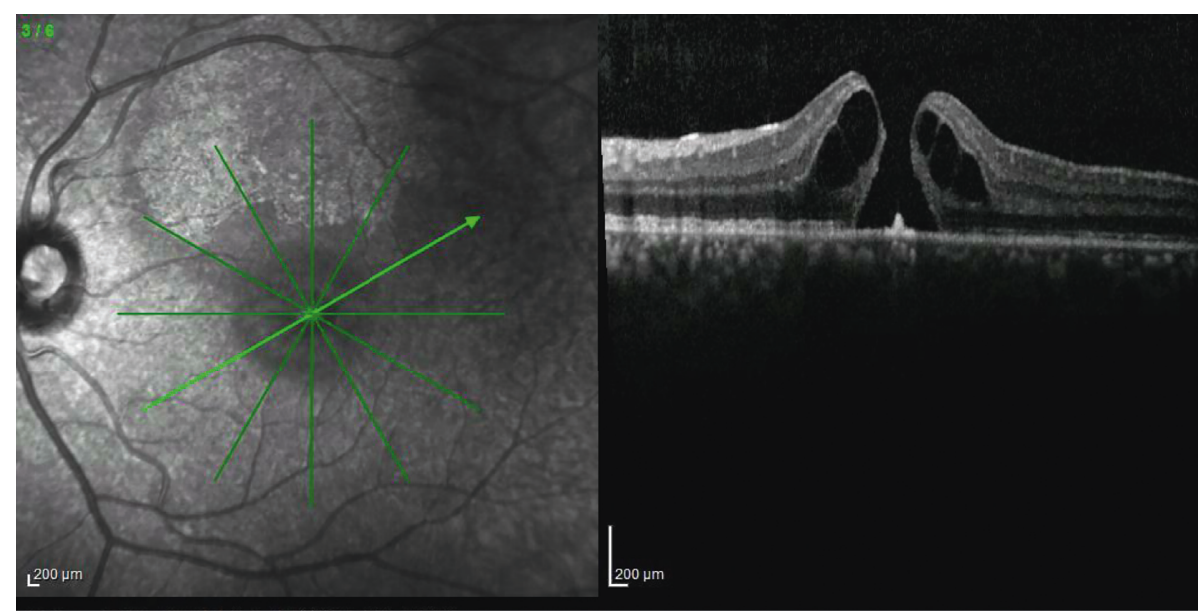

(a)

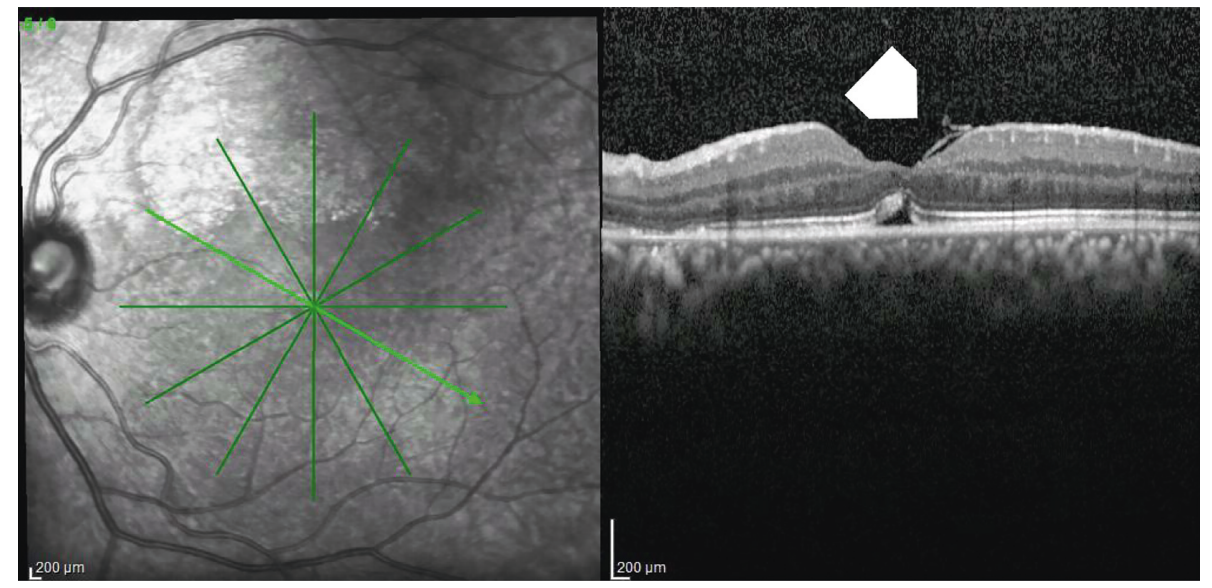

(b)

FIgURE 3: (a) Radial scan OCT image of the left eye of a 16-year-old male. The patient was hit by a closed fist at 15 days. The MLD of the FTMH was $359 \mu \mathrm{m}$. His BCVA was 0.1 Snellen. Note the cystic thickening at the edges of the hole with typical pregnant drawbridge appearance. (b) Radial scan OCT of the same eye approximately 2.5 months after PPV and IFT showing U-type closure. Note the residual subfoveal neurosensory detachment, fully restored ELM, and partially restored IS/OS layer. The ILM flap was seen folded on itself into the macular hole (white arrowhead). His final BCVA was 0.4 Snellen.

TABle 2: Postoperative anatomical and functional outcomes.

\begin{tabular}{lcc}
\hline Postoperative outcome & Group I & Group II \\
& $N=28$ & $N=12$ \\
\hline Closure type & & \\
U-type & $6(21.4 \%)$ & $6(50 \%)$ \\
V-type & $15(53.5 \%)$ & $5(42 \%)$ \\
W-type & $7(25 \%)$ & $1(8.3 \%)$ \\
\hline Foveal microstructure & & \\
Fully restored & $3(11 \%)$ & $1(8.3 \%)$ \\
Partially restored & $5(18 \%)$ & - \\
Not restored & $13(46.4 \%)$ & $10(83.3 \%)$ \\
Persistent open hole & $7(25 \%)$ & $1(8.3 \%)$ \\
\hline BCVA logMAR (Snellen) & & \\
Mean & $1(20 / 200)$ & $0.7(20 / 100)$ \\
$>1(<20 / 200)$ & $9(32 \%)$ & $1(8.3 \%)$ \\
$1-0.4(20 / 50-20 / 200)$ & $15(53.5 \%)$ & $11(92 \%)$ \\
$<0.4(>20 / 50)$ & $4(14 \%)$ & - \\
\hline Cataract formation & $12(43 \%)$ & $3(25 \%)$ \\
\hline
\end{tabular}

BCVA, best-corrected visual acuity; logMAR, logarithm of the minimal angle of resolution. traumatic macular hole secondary to retinal hemorrhages in shaken baby syndrome. The authors performed PPV and ILM peel for 4 patients and reported macular hole closure rate of $75 \%$. The mean macular hole diameter was $700 \mu \mathrm{m}$. A more recent retrospective comparative case series by Ghoraba et al. [23] compared the use of C3F8 and silicone oil in 2 groups of patients who underwent PPV and ILM peel for traumatic macular holes. The authors reported primary closure rate of $81.8 \%$ and final closure rate of $90.9 \%$ after reoperation, although no information was provided on the preoperative macular hole diameter. The authors mentioned that the overall mean improvement of BCVA was 3 and 4 lines in the silicone oil and C3F8 groups, respectively. There was no information on subgroup stratification in terms of lines of vision gained, lost, or unchanged and how did that correlate with foveal microstructure (Table 6).

To our knowledge, the present study is the first report comparing the outcome of ILM peel technique and IFT in traumatic macular holes. The paucity of literature on outcomes of comparison of both techniques in this particular 
TABLE 3: Correlation between surgical techniques (PPV with ILM peel versus PPV with IFT).

\begin{tabular}{|c|c|c|c|c|c|c|}
\hline \multirow{2}{*}{ Groups } & \multicolumn{2}{|c|}{ Postoperative BCVA } & \multicolumn{2}{|c|}{ Foveal microstructure } & \multicolumn{2}{|c|}{ Closure type } \\
\hline & Mean & SE & Mean & SE & Mean & SE \\
\hline Without IFT & 0.96 & 0.07 & 2.85 & 0.17 & 2.03 & 0.13 \\
\hline With IFT & 0.7 & 0.07 & 2.9 & 0.19 & 1.58 & 0.19 \\
\hline$T$ value & \multicolumn{2}{|c|}{0.19} & \multicolumn{2}{|c|}{0.2} & \multicolumn{2}{|c|}{1.91} \\
\hline$P$ value & \multicolumn{2}{|c|}{0.02} & \multicolumn{2}{|c|}{0.84} & \multicolumn{2}{|c|}{0.05} \\
\hline
\end{tabular}

BCVA, best-corrected visual acuity; IFT, ILM flap technique; ILM, internal limiting membrane; PPV, pars plana vitrectomy; SE, standard error of the mean.

TABLE 4: Group I: statistical correlation between preoperative parameters and postoperative anatomic and functional outcomes.

\begin{tabular}{|c|c|c|c|c|}
\hline & & Postoperative BCVA (logMAR) & Closure type & Foveal microstructure \\
\hline \multirow{3}{*}{ Preoperative BCVA (logMAR) } & Pearson correlation & $0.39^{*}$ & 0.12 & 0.07 \\
\hline & Sig. (2-tailed) & 0.039 & 0.53 & 0.71 \\
\hline & $N$ & 28 & 28 & 28 \\
\hline \multirow{3}{*}{ Preoperative MLD $(\mu \mathrm{m})$} & Pearson correlation & $0.52^{* *}$ & 0.26 & 0.28 \\
\hline & Sig. (2-tailed) & 0.004 & 0.17 & 0.14 \\
\hline & $N$ & 28 & 28 & 28 \\
\hline \multirow{3}{*}{ Disease duration (months) } & Pearson correlation & 0.12 & -0.11 & -0.05 \\
\hline & Sig. (2-tailed) & 0.53 & 0.54 & 0.78 \\
\hline & $N$ & 28 & 28 & 28 \\
\hline
\end{tabular}

BCVA, best-corrected visual acuity; logMAR, logarithm of the minimal angle of resolution; $\mu \mathrm{m}$, micron; MLD, minimum linear diameter. ${ }^{*}$ Correlation is significant at the 0.05 level (2-tailed). ${ }^{* *}$ Correlation is significant at the 0.01 level (2-tailed).

TABLE 5: Group II: statistical correlation between preoperative parameters and postoperative anatomic and functional outcomes.

\begin{tabular}{|c|c|c|c|c|}
\hline & & Postoperative BCVA (logMAR) & Closure type & Foveal microstructure \\
\hline \multirow{3}{*}{ Preoperative BCVA (logMAR) } & Pearson correlation & 0.27 & -0.17 & 0.23 \\
\hline & Significance (2-tailed) & 0.38 & 0.57 & 0.45 \\
\hline & $N$ & 12 & 12 & 12 \\
\hline \multirow{3}{*}{ Preoperative MLD $(\mu \mathrm{m})$} & Pearson correlation & -0.14 & 0.19 & 0.48 \\
\hline & Significance (2-tailed) & 0.96 & 0.54 & 0.11 \\
\hline & $N$ & 12 & 12 & 12 \\
\hline \multirow{3}{*}{ Disease duration (months) } & Pearson correlation & 0.09 & -0.03 & 0.07 \\
\hline & Significance (2-tailed) & 0.77 & 0.91 & 0.81 \\
\hline & $N$ & 12 & 12 & 12 \\
\hline
\end{tabular}

BCVA, best-corrected visual acuity; logMAR, logarithm of the minimal angle of resolution; $\mu \mathrm{m}$, micron; MLD, minimum linear diameter; $N$, number.

category of macular holes is a significant deterrent to purposeful validation of our findings in the current study. Moreover, most of the published data on traumatic macular holes were derived from retrospective studies and case reports. Nevertheless, we could compare our results to other studies that compared both techniques in other categories of macular holes that are categorized as recalcitrant macular holes such as large holes and myopic macular holes. Table 7 summarizes the outcome of different studies that compared PPV and ILM peel versus inverted ILM flap technique in treating different recalcitrant macular holes in comparison with the outcome of the present study.

In summary, the results of the present study demonstrated that IFT is significantly superior to ILM peel in terms of more anatomical macular hole closure and final BCVA. It is worthy of note that, in the IFT group in the present study, we adopted Casini et al. [28] modification of the classic inverted ILM flap technique described by Michalewska et al.
[24] in the sense that we did not attempt to invert the ILM flap and fold it inside the hole to avoid damaging the RPE at the bed of the hole. The rationale of our modified approach is that shearing of the foot plates of the Müller cells during ILM peel and residual attachment of the ILM flap to the hole edges would suffice to incite glial cell proliferation and that eventually fills up the macular hole and promotes its closure $[11,24,31]$.

Limitations of the current study included its retrospective design that dictated inhomogeneity of the compiled data under the ILM peel group and the IFT group, in terms of number of eyes recruited, macular hole MLD, and duration of follow-up. For instance, $50 \%$ of patients in group I had baseline MLD $>800 \mu \mathrm{m}$ versus $8.3 \%$ in group II. Given that our statistical analysis revealed significant correlation between preoperative MLD and final BCVA in group I, this could mean that group I patients had worse visual outcome due to initial much larger MLD. However, we could argue that statistical analysis revealed no significant correlation 
TABLE 6: Review of studies on PPV and ILM peel for traumatic macular hole.

\begin{tabular}{|c|c|c|c|c|}
\hline Author & $\begin{array}{c}\text { No. of } \\
\text { eyes }\end{array}$ & Surgical technique & $\begin{array}{c}\text { Anatomical closure, } \\
\text { no. }(\%)\end{array}$ & $\begin{array}{l}\text { Functional outcome } \\
\text { (mean final BCVA) }\end{array}$ \\
\hline Kuhn et al. [22] & 17 & $\begin{array}{l}\text { PPV-ILM peel } \\
\text { SF6 }\end{array}$ & $17(100)$ & 6 lines \\
\hline Johnson et al. [14] & 25 & $\begin{array}{c}\text { PPV-ILM peel (3 cases) } \\
\text { C3F8 } \\
\text { Autologous serum (12 cases) }\end{array}$ & $24(96)$ & $\begin{array}{l}\geq 2 \text { lines in } 84 \% \\
\text { of cases }\end{array}$ \\
\hline Ou et al. [17] & 4 & $\begin{array}{c}\text { PPV } \\
\text { ILM peel (4 cases) } \\
\text { SO, air, C3F8, no tamponade }(1 \text { case })\end{array}$ & $3(75)$ & Poor visual outcome \\
\hline Ghoraba et al. [23] & 22 & $\begin{array}{c}\text { PPV-ILM peel-SO (9 cases) } \\
\text { PPV-ILM peel } \\
\text { C3F8 (14 cases }) \\
\end{array}$ & $\begin{array}{l}81.8 \% \text { primary closure, } \\
90.9 \% \text { after reoperation }\end{array}$ & $\begin{array}{l}3 \text { lines (SO group), } \\
4 \text { lines (C3F8 group) }\end{array}$ \\
\hline $\begin{array}{l}\text { Current study, } 2018 \text { (first } \\
\text { comparison between } \\
\text { ILM peel technique and IFT) }\end{array}$ & 40 & $\begin{array}{l}\text { PPV-ILM-C2F6 } \\
\text { PPV-IFT-C2F6 }\end{array}$ & $\begin{array}{l}75 \% \\
92 \%\end{array}$ & $\begin{array}{l}2.5 \text { lines } \\
5 \text { lines }\end{array}$ \\
\hline
\end{tabular}

BCVA, best-corrected visual acuity; C2F6, hexafluoroethane; C3F8, octafluoropropane; IFT, ILM flap technique; ILM, internal limiting membrane; no., number; PPV, pars plana vitrectomy; SF6, sulfur hexafluoride; SO, silicone oil.

TABLE 7: Review of studies on PPV and ILM peel versus PPV and inverted ILM flap for different types of macular holes.

\begin{tabular}{|c|c|c|c|c|c|}
\hline Author & Macular hole type & Surgical technique & $\begin{array}{l}\text { No. of } \\
\text { eyes }\end{array}$ & $\begin{array}{l}\text { Anatomical } \\
\text { closure }(\%)\end{array}$ & $\begin{array}{l}\text { Functional outcome } \\
\text { (mean final BCVA) }\end{array}$ \\
\hline \multirow{2}{*}{$\begin{array}{l}\text { Michalewska et al. } \\
\text { [24] }\end{array}$} & \multirow[t]{2}{*}{ Idiopathic } & PPV, ILM peel, air & 51 & $88 \%$ & $\begin{array}{l}\text { (Pre-op 0.12)-(post-op 0.17); } \\
\text { Snellen }\end{array}$ \\
\hline & & PPV, inverted ILM flap, air & 50 & $98 \%$ & (Pre-op 0.07)-(post-op 0.2); Snellen \\
\hline Chen et al. [25] & Idiopathic & PPV, inverted ILM flap, C3F8 & 8 & $100 \%$ & (Pre-op 1.3)-(post-op 0.6); logMAR \\
\hline \multirow{3}{*}{ Sasaki et al. [26] } & \multirow{3}{*}{$\begin{array}{c}\text { Macular } \\
\text { hole-associated } \\
\text { retinal detachment }\end{array}$} & PPV, ILM peel & 9 & $55.5 \%$ & $\begin{array}{c}\text { (Pre-op 1.00)-(post-op 1.02); } \\
\text { logMAR }\end{array}$ \\
\hline & & PPV, inverted ILM flap & 6 & $100 \%$ & $\begin{array}{c}\text { (Pre-op 1.04)-(post-op 0.6); } \\
\text { logMAR }\end{array}$ \\
\hline & & $\mathrm{C} 3 \mathrm{~F} 8$ or $\mathrm{SF} 6$ & & & \\
\hline \multirow{2}{*}{ Mete et al. [27] } & \multirow{2}{*}{ Myopic } & PPV, ILM peel, SF6 & 36 & $61 \%$ & $\begin{array}{c}\text { (Pre-op 0.6) -(post-op 0.58); } \\
\text { logMAR }\end{array}$ \\
\hline & & PPV, inverted ILM flap, SF6 & 34 & $94 \%$ & $\begin{array}{c}\text { (Pre-op 0.7)-(post-op 0.39); } \\
\text { logMAR }\end{array}$ \\
\hline \multirow{2}{*}{ Casini et al. [28] } & \multirow{2}{*}{ Idiopathic } & PPV, inverted ILM flap, SF6 & 41 & $97.6 \%$ & $\begin{array}{c}\text { (Pre-op 20/120)-(Post-op 20/30) } \\
\text { Snellen }\end{array}$ \\
\hline & & $\begin{array}{l}\text { PPV, modified inverted ILM flap, } \\
\text { SF6 }\end{array}$ & 40 & $97.5 \%$ & $\begin{array}{c}\text { (Pre-op 20/132)-(Post-op 20/35); } \\
\text { Snellen }\end{array}$ \\
\hline \multirow{2}{*}{ Kannan et al. [29] } & \multirow{2}{*}{ Idiopathic } & PPV, ILM peel, SF6 & 30 & $70 \%$ & 1.4 lines \\
\hline & & PPV, inverted ILM flap, SF6 & 30 & $90 \%$ & 2.1 lines \\
\hline \multirow{3}{*}{ Rizzo et al. [30] } & \multirow{3}{*}{ Idiopathic, myopic } & PPV, ILM peel & 300 & $78.75 \%$ & $\begin{array}{c}\text { (Pre-op 0.77)-(post-op 0.52); } \\
\text { logMAR }\end{array}$ \\
\hline & & PPV, inverted ILM flap & 320 & $91.93 \%$ & $\begin{array}{c}\text { (Pre-op 0.74)-(post-op 0.43); } \\
\text { logMAR }\end{array}$ \\
\hline & & $\mathrm{C} 3 \mathrm{~F} 8$ or SF6 & & & \\
\hline \multirow{2}{*}{ Current study, 2018} & \multirow{2}{*}{ Traumatic } & PPV, ILM, C2F6 & 28 & $75 \%$ & 2.5 lines \\
\hline & & PPV, IFT, C2F6 & 12 & $92 \%$ & 5 lines \\
\hline
\end{tabular}

BCVA, best-corrected visual acuity; C2F6, hexafluoroethane; C3F8, octafluoropropane; IFT, ILM flap technique; ILM, internal limiting membrane; logMAR, logarithm of minimum angle of resolution; no., number; PPV, pars plana vitrectomy; SF6, sulfur hexafluoride.

between baseline MLD and macular hole closure or restoration of foveal microstructure in both groups. By extrapolation, the cause of worse final BCVA in group I was related to inferior macular hole closure rate rather than baseline MLD, which adds to the strength of our results as it corroborates the higher efficacy of IFT.

\section{Conclusion}

PPV and IFT is associated with significantly superior anatomic and functional outcomes of traumatic FTMH compared to PPV and ILM peel. Randomized comparative clinical trials focused on surgical management of traumatic 
FTMH are needed to assert noninferiority or equivalence of PPV and IFT to emerging surgical alternatives before recommending it as a standard surgical approach to traumatic FTMH.

\section{Data Availability}

The statistical data used to support the findings of this study are included within the article. The data collected from history taking and clinical examination of patients recruited in the current study are confidential. Access to these data is restricted by Magrabi Eye Hospital, Tanta, Egypt, in accordance with the hospital's patients' data protection policy. Data are available for researchers who meet the criteria for access to confidential data through contacting the hospital's medical director Professor Hammouda Ghoraba.

\section{Additional Points}

Traumatic FTMH acquired notoriety of morbid outcome due to sequelae of trauma as tissue loss and retinal atrophy. Despite a myriad of surgical maneuvers, there is no current consensus on the ideal surgical technique. IFT is a promising approach that would improve the final outcome compared to ILM peel.

\section{Disclosure}

The study was conducted in Magrabi Eye Hospital, Tanta, Egypt. The manuscript involves the use of triamcinolone acetonide (TA) as an adjuvant for ILM peel. Currently, TA is used as an off-label ocular therapeutic agent that is not approved by FDA.

\section{Conflicts of Interest}

None of the authors has competing financial, professional, or personal interest that might have influenced the performance or presentation of the work described in this manuscript. None of the authors has commercial associations that might pose a conflict of interest in connection with the submitted article. None of the authors has proprietary interest in any of the materials discussed in the study.

\section{Supplementary Materials}

Supplemental digital content 1 . Video demonstrates the IFT using TA.mp4. (Supplementary Materials)

\section{References}

[1] N. E. Kelly and R. T. Wendel, "Vitreous surgery for idiopathic macular holes," Archives of Ophthalmology, vol. 109, no. 5, pp. 654-659, 1991.

[2] R. T. Wendel, A. C. Patel, N. E. Kelly, T. C. Salzano, J. W. Wells, and G. D. Novack, "Vitreous surgery for macular holes," Ophthalmology, vol. 100, no. 11, pp. 1671-1676, 1993.

[3] J. D. M. Gass, "Idiopathic senile macular hole," Archives of Ophthalmology, vol. 106, no. 5, pp. 629-639, 1988.
[4] J. D. M. Gass, "Reappraisal of biomicroscopic classification of stages of development of a macular hole," American Journal of Ophthalmology, vol. 119, no. 6, pp. 752-759, 1995.

[5] J. D. M. Gass, "Müller cell cone, an overlooked part of the anatomy of the fovea centralis," Archives of Ophthalmology, vol. 117 , no. 6, pp. 821-823, 1999.

[6] A. Gaudric and R. Tadayoni, "Macular hole," in Retina, S. J. Ryan, Ed., vol. 5, pp. 1962-1978, Saunders, Philadelphia, PA, USA, 2013.

[7] J. W. Berger and A. J. Brucker, "The magnitude of the bubble buoyant pressure," Retina, vol. 18, no. 1, pp. 84-86, 1998.

[8] C. Eckardt, U. Eckardt, S. Groos, L. Luciano, and E. Reale, "Entfernung der membrana limitans interna bei makulalöchern," Der Ophthalmologe, vol. 94, no. 8, pp. 545-551, 1997.

[9] C. K. Spiteri, N. Lois, N. Scott et al., "Vitrectomy with internal limiting membrane (ILM) peeling versus vitrectomy with no peeling for idiopathic full-thickness macular hole (FTMH)," Cochrane Database of Systematic Reviews, no. 6, article CD009306, 2013.

[10] M. Parravano, F. Giansanti, C. M. Eandi, Y. C. Yap, S. Rizzo, and G. Virgili, "Vitrectomy for idiopathic macular hole," Cochrane Database of Systematic Reviews, vol. 12, no. 5, article CD009080, 2015.

[11] W. E. Smiddy, W. Feuer, and G. Cordahi, "Internal limiting membrane peeling in macular hole surgery," Ophthalmology, vol. 108, no. 8, pp. 1471-1476, 2001.

[12] J. Huang, X. Liu, Z. Wu, and S. Sadda, "Comparison of fullthickness traumatic macular holes and idiopathic macular holes by optical coherence tomography," Graefe's Archive for Clinical and Experimental Ophthalmology, vol. 248, no. 8, pp. 1071-1075, 2010.

[13] D. Shukla, "Secondary macular holes: when to jump in and when to stay out," Expert Review of Ophthalmology, vol. 8, no. 5, pp. 437-446, 2013.

[14] R. N. Johnson, H. R. McDonald, H. Lewis et al., "Traumatic macular hole," Ophthalmology, vol. 108, no. 5, pp. 853-857, 2001.

[15] E. D. Weichel and M. H. Colyer, "Traumatic macular holes secondary to combat ocular trauma," Retina, vol. 29, no. 3, pp. 349-354, 2009.

[16] N. Kunjukunju, A. Navarro, S. Oliver et al., "Bilateral macular hole formation secondary to sclopetaria caused by shockwaves transmitted by a posterior vector: case report," BMC Ophthalmology, vol. 10, no. 6, 2010.

[17] J. I. Ou, D. M. Moshfegi, K. Tawansy, and J. E. Sears, "Macular hole in the shaken baby syndrome," Archives of Ophthalmology, vol. 124, no. 6, pp. 913-915, 2006.

[18] T. Rossi, B. Boccassini, L. Esposito et al., "The pathogenesis of retinal damage in blunt eye trauma: finite element modeling," Investigative Opthalmology \& Visual Science, vol. 52, no. 7, pp. 3994-4002, 2011.

[19] M. Fiorentzis, B. Seitz, and A. Viestenz, "Traumatisches makulaforamen," Klinische Monatsblätter für Augenheilkunde, vol. 235, no. 7, 2018.

[20] M. S. Ip, B. J. Baker, J. S. Duker et al., "Anatomical outcomes of surgery for idiopathic macular hole as determined by optical coherence tomography," Archives of Ophthalmology, vol. 120, no. 1, pp. 29-35, 2002.

[21] M. Imai, H. Iijima, T. Gotoh, and S. Tsukahara, "Optical coherence tomography of successfully repaired idiopathic macular holes," American Journal of Ophthalmology, vol. 128, no. 5, pp. 621-627, 1999. 
[22] F. Kuhn, R. Morris, V. Mester, and C. Witherspoon, "Internal limiting membrane removal for traumatic macular holes," Ophthalmic Surg Lasers, vol. 32, pp. 308-315, 2001.

[23] H. H. Ghoraba, A. F. Ellakwa, and A. A. Ghali, "Long term result of silicone oil versus gas tamponade in the treatment of traumatic macular holes," Clinical Ophthalmology, vol. 2012, no. 6, pp. 49-53, 2012.

[24] Z. Michalewska, J. Michalewski, R. A. Adelman, and J. Nawrocki, "Inverted internal limiting membrane flap technique for large macular holes," Ophthalmology, vol. 117, no. 10, pp. 2018-2025, 2010.

[25] Z. Chen, C. Zhao, J.-J. Ye, X.-Q. Wang, and R.-F. Sui, "Inverted internal limiting membrane flap technique for repair of large macular holes," Chinese Medical Journal, vol. 129, no. 5, pp. 511-517, 2016.

[26] H. Sasaki, A. Shiono, J. Kogo et al., "Inverted internal limiting membrane flap technique as a useful procedure for macular hole-associated retinal detachment in highly myopic eyes," Eye, vol. 31, no. 4, pp. 545-550, 2017.

[27] M. Mete, A. Alfano, M. Guerriero et al., "Inverted internal limiting membrane flap technique versus complete internal limiting membrane removal in myopic macular hole surgery," Retina, vol. 37, no. 10, pp. 1923-1930, 2017.

[28] G. Casini, M. Mura, M. Figus et al., "Inverted internal limiting membrane flap technique for macular hole surgery without extra manipulation of the flap," Retina, vol. 37, no. 11, pp. 2138-2144, 2017.

[29] N. B. Kannan, P. Kohli, H. Parida et al., "Comparative study of inverted internal limiting membrane (ILM) flap and ILM peeling technique in large macular holes: a randomizedcontrol trial," BMC Ophthalmology, vol. 18, no. 1, p. 177, 2018.

[30] S. Rizzo, R. Tartaro, F. Barca, T. Caporossi, D. Bacherini, and F. Giansanti, "Internal limiting membrane peeling versus inverted flap technique for treatment of full-thickness macular holes," Retina, vol. 38, no. S1, pp. S73-S78, 2018.

[31] K. Yamashiro, E. Kinoshita-Nakano, T. Ota, Y. Jingami, I. Nakata, and H. Hayashi, "Floating flap of internal limiting membrane in myopic macular hole surgery," Graefe's Archive for Clinical and Experimental Ophthalmology, vol. 256, no. 4, pp. 693-698, 2018. 


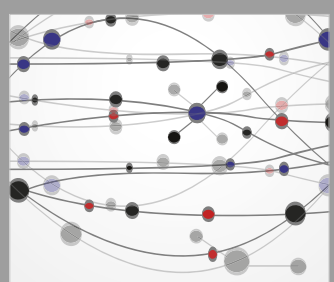

The Scientific World Journal
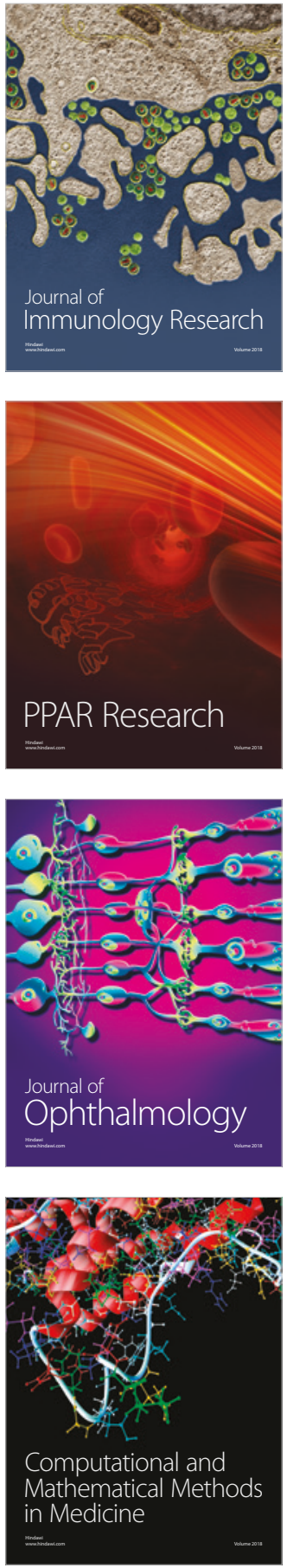

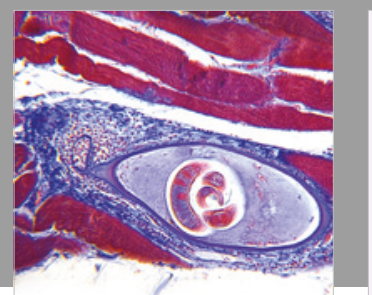

Gastroenterology Research and Practice

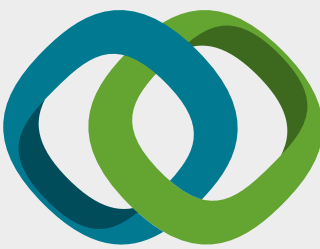

\section{Hindawi}

Submit your manuscripts at

www.hindawi.com
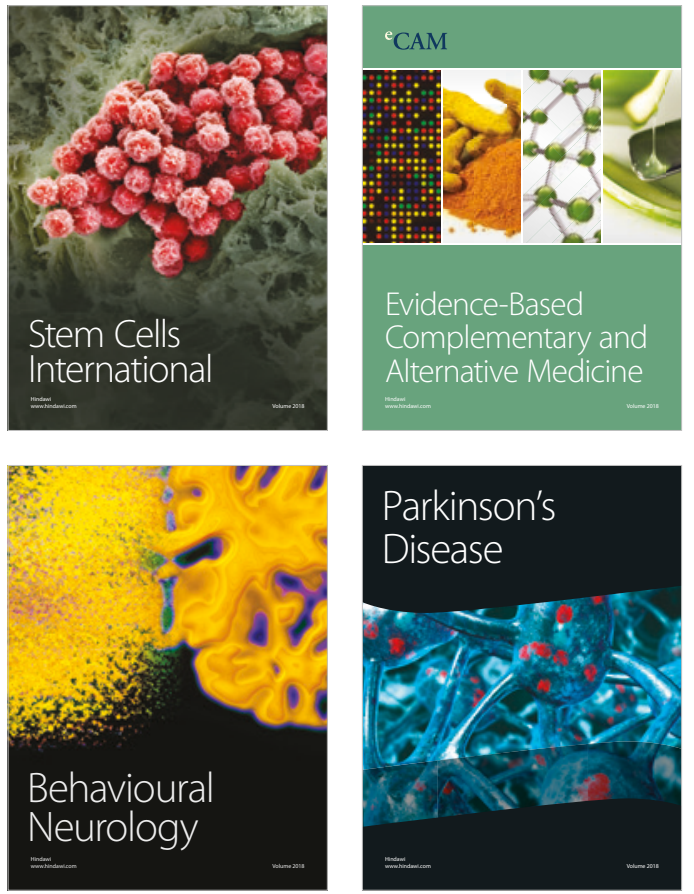

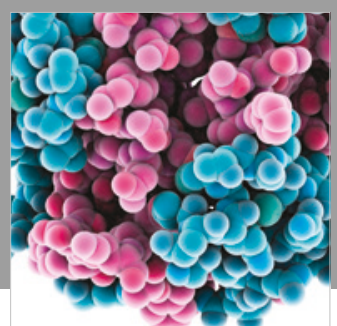

ournal of

Diabetes Research

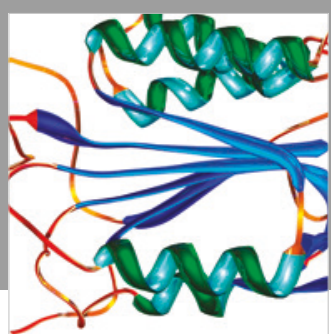

Disease Markers
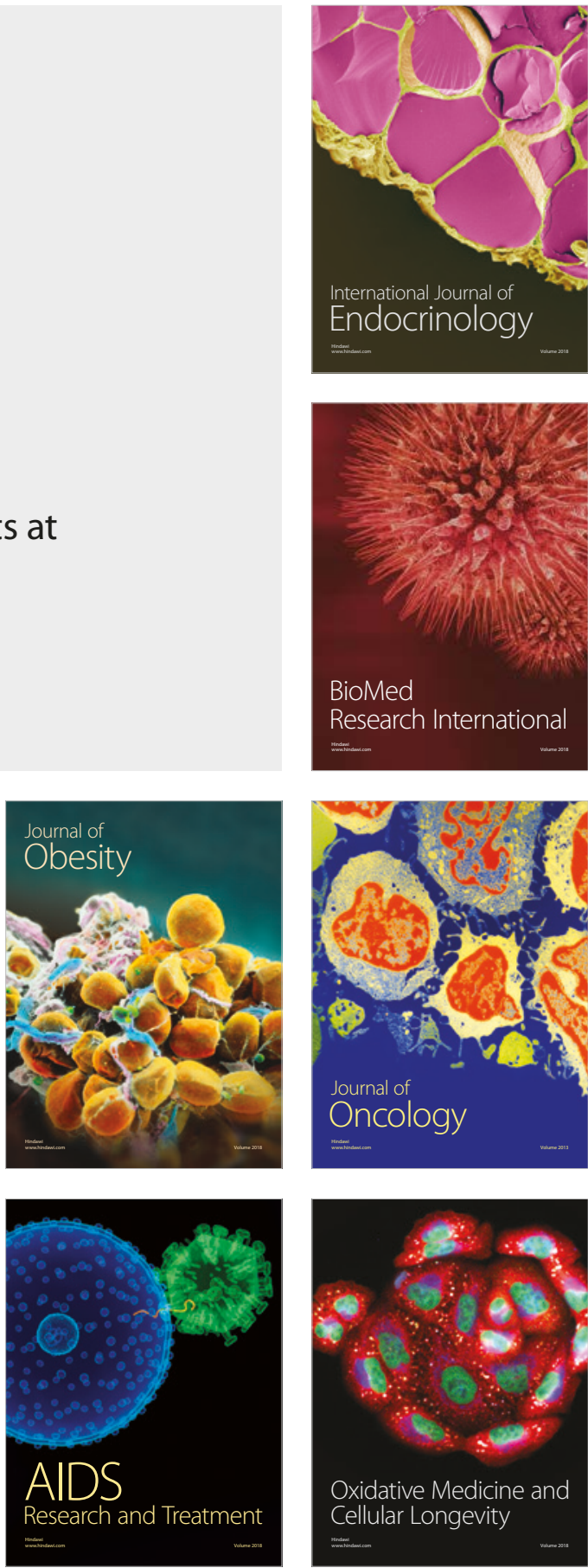